\title{
Effect of Lactic Acid Bacteria-Fermented Soy Milk Extract (LEX) on Urinary 3-Indoxyl Sulfate in Japanese Healthy Adult Women: An Open-Label Pilot Study
}

Nutrition and Dietary Supplements

\section{Mina Fukuchi ${ }^{1}$ \\ Takanori Yasutake ${ }^{2}$ \\ Miho Matsumoto ${ }^{2}$ \\ Ryohei Mizuno ${ }^{3}$ \\ Koichiro Fujita ${ }^{4}$ \\ Yasuhiro Sasuga'}

'Hachioji Center for Research \& Development, B\&S Corporation Co., Ltd., Hachoji-shi, Tokyo, Japan; ${ }^{2}$ Healthcare Systems Co., Ltd., Nagoya-shi, Aichi, Japan; ${ }^{3}$ B\&S Corporation Co, Ltd., Tokyo, Japan; ${ }^{4}$ Tokyo Medical and Dental University, Tokyo, Japan
Correspondence: Yasuhiro Sasuga Hachioji Center for Research and Development, B\&S Corporation Co., Ltd., I-3I-I Nanakuni, Hachioji-Shi, Tokyo 192-0919, Japan

Tel $+8 \mid 42-638-8915$

Fax +81 42-638-8916

Email y-sasuga@bandscorp.jp
Objective: For the general population, the intestinal microbiota and its environment, including its metabolites, are important not only for disease prevention but also for overall quality of life. A convenient method to monitor the intestinal environment is to measure levels of urinary 3-indoxyl sulfate (3-IS). This approach has been used for several years. This study aims to clarify the efficacy of a lactic acid bacteria (LAB) dietary supplement combined with multi-strain LAB-fermented soy milk (LEX) extract in healthy, adult women with high urinary 3-IS levels.

Materials and Methods: Japanese women aged 30-50 years with high urinary 3-IS levels and a tendency toward constipation were enrolled in this study. Each of them received $10 \mathrm{~mL}$ of a LEX-containing dietary supplement daily for 4 weeks. The primary outcome was changes in urinary 3-IS levels, and the secondary ones included the amount of defecation and more subjective symptoms of their physical condition, such as the Constipation Assessment Scale (CAS).

Results: Thirty participants were enrolled, and all completed the study. Every participant showed a statistically significant improvement in their level of urinary 3-IS after ingesting the test article for 4 weeks. Any changes in urinary 3-IS also showed a high correlation to initial 3-IS levels. Moreover, CAS scores and other subjective symptoms improved after ingestion of the LEX-containing dietary supplement. The level of urinary 3-IS showed a modest degree of correlation with the score of overall physical condition, including CAS (Pearson's $\mathrm{r}=0.21 ; p<0.05$ ).

Conclusion: These findings suggest that orally ingested LEX improves urinary 3-IS levels as an index of the health of the intestinal environment and that monitoring urinary 3-IS levels is effective in assessing changes in the intestinal environment and overall physical condition. Keywords: lactic acid bacteria, soybean, 3-indoxyl sulfate, intestinal environment, intestinal microbiota, postbiotics

\section{Introduction}

The gut not only functions as the digestive organ for food and the absorption of nutrients but also helps maintain systemic immunity, supports regulation of the central nervous system, and aids in detoxification. Mounting evidence indicates that the functions of the gut are affected by its environment, which in turn is affected by factors such as diet and commensal bacteria. The gut environment's health is also 
related to the development of several diseases, eg, allergies and inflammation. The use of next-generation sequencing techniques has greatly expanded our knowledge of the microbiota composition and its relationship to disease. ${ }^{1}$ Recently, there have been many debates on what exactly constitutes a healthy gut microbiota composition. Current evidence supports the idea that healthy individuals share a common core of gut microbial genes mainly belonging to the Firmicutes and Bacteroidetes phyla. ${ }^{2}$ On the other hand, there have been reports that show that a high variability in gut microbiota composition is common among healthy individuals. Thus, it has become clear that each individual carries a variable microbiome. ${ }^{3}$

Diet is the main factor that determines the population of human microbiota, as dietary components act as substrates for microbial metabolism, thus forming the microbiome composition and function. ${ }^{4}$ Microbiota composition has been linked to disease due to its ability to modulate specific metabolites and signaling pathways. For example, a gut microbiota-dependent metabolite called trimethylamine $\mathrm{N}$-oxide (TMAO) has been reported to cause atherosclerosis and thrombosis. ${ }^{5}$ As TMAO demonstrates, it is increasingly clear that the microbiota and its metabolites are important cofactors of host physiology and pathophysiology. ${ }^{6-8}$

Changes in the intestinal environment, including its metabolites, are important not only for disease prevention but also for quality of life in the general population. Conditions such as bad skin, constipation, diarrhea, and poor overall health can be related to the intestinal environment. Using expensive analysis methods, such as next-generation sequencing and metabolome analysis, is unrealistic for such physical condition changes in normal life. An easy and cost-effective way to observe changes in the intestinal environment and bacteria is needed.

A little old study found that several urinary metabolites (eg, indole derivatives, phenols, and amines, which originate from amino acids) may represent the condition of the intestinal environment. 3-indoxyl sulfate (3-IS) is a metabolite of dietary L-tryptophan (Trp) and acts as a cardiotoxin and uremic toxin. The intestinal microbiota metabolizes Trp into indole, which is then absorbed by the intestine and then further converted into 3-IS by the liver. ${ }^{9}$ Urinary 3-IS has been used as a screening tool for microbiota dysbiosis, small intestinal bacterial overgrowth, constipation, malabsorption, and intestinal mucosal permeability. ${ }^{9-13}$ The level of 3-IS can therefore be used for simple observations of the intestinal environment, which is easily and inexpensively measured using the colorimetric method on a urine sample.
The health benefits of lactic acid bacteria (LAB)-based products have been recognized worldwide. LAB fermentation is known to facilitate the digestion of foods or increase the bioavailability of nutrients in foods. Various types of LAB (as probiotics) and LAB-fermented food products have been developed and present benefits to both humans and animals, such as an improvement of the nutritional value of food, increased ability to prevent infections, alleviation of lactose-intolerance symptoms, modulation of specific/nonspecific immune responses, control of serum cholesterol levels, and reduction of colonic disease risk. ${ }^{14,15}$ The soybean has long been used as fermentation material, particularly in East Asia, for products such as soy sauce, miso, natto, and tempeh. Several studies on the fermentation of soybeans (and soy milk) using LAB report on the physiological function of these products. However, most of these reports focus on the hepatic functions of LAB-fermented soy, such as the metabolism of lipids like cholesterol and triglycerides, because soybean is known to improve lipid metabolism. ${ }^{16-21}$

Another study demonstrated that the ingestion of LAB dietary supplements derived from the extract of multi-strain LAB-fermented soy milk (LEX) can prevent colon cancer and activate intestinal immunity. ${ }^{22,23}$ Preliminary studies have also shown how LEX effects human colon polyps, intestinal flora, and periodontal disease. ${ }^{24-26}$ The positive results are based on how the LEX supplement improves the intestinal environment and microbiota composition, although verification is required. "Postbiotics" is also a relatively new term in the "-biotics" field, after probiotics and prebiotics. Postbiotics are functional, bioactive compounds produced in the matrix during fermentation and may improve human health. ${ }^{27,28}$ LEX is also a postbiotic material that uses the soybean as a substrate. Studies on LEX could contribute to the expansion of the postbiotics field.

In this pilot study, we used urinary 3-IS levels to assess the intestinal environment of our subjects and to evaluate the effects of LEX on the intestinal environment and microbiota metabolism. Changes in urinary 3-IS before and after the ingestion of LEX were investigated in healthy women with high urinary 3-IS levels. In addition, abdominal symptoms and other daily physical condition changes were investigated.

\section{Materials and Methods}

\section{Study Design and Participants}

This study was an open-label study evaluating the effects of LEX on urinary 3-IS. Secondary endpoints were any 
changes in subjective symptoms related to defecation and overall physical condition. This study enrolled Japanese women aged 30-50 years who had a tendency to become constipated. Sixty applicants were screened and 30 participants with high urinary 3-IS levels were enrolled. Those who had chronic illnesses or were allergic to soybeans, a raw material of the test substance, were excluded. Informed consent was obtained from each subject and ethical approval was obtained from the Ethics Committee of Healthcare Systems Co., Ltd. (registration number: 2001, Tokyo, Japan). The study was conducted according to the Declaration of Helsinki and was registered at the University Hospital Medical Information Network (ID UMIN000039276).

\section{Test Article}

The test article was a commercially available LAB dietary supplement (brand name LACTIS ${ }^{\mathrm{TM}}$ ) that was derived from multi-strain LAB-fermented soy milk (LEX) extract, obtained from B\&S Corporation Co., Ltd. (Tokyo, Japan). Sixteen strains of LAB (species Lactobacillus brevis, Lactobacillus casei, and Lactobacillus plantarum) were used for the fermentation of the soy milk, then the extract of the fermented material was used in the final product.

\section{Procedure}

Participants were recruited on a dedicated website, and 60 constipated and test-matched individuals were selected using a preliminary questionnaire. Of these, 30 participants were enrolled based on their urinary 3-IS levels. The enrolled participants were provided with the test article, a diary, questionnaires, and a urine collection kit and were instructed to ingest the test article once daily for 4 weeks.

The primary outcome was to measure levels of urinary 3-IS. These levels were measured three times in total, before the start of the experiment, and 2 and 4 weeks after starting intake of the test article using a quantitative indican assay kit (BioAssay Systems, CA, USA). This is an improved version of the Curzon and Walsh method with a colorimetric assay, corrected by creatinine $(\mathrm{Cr})$ concentration, using mid-morning urine. To quantify the $\mathrm{Cr}$ in urine, an automatic analyzer (Accute RX, Canon Medical Systems, Tochigi, Japan) and a Cr assay kit (CicaLiquid-N CRE, Kanto Kagaku, Tokyo, Japan) were used.

To track secondary outcomes, a daily diary and three questionnaire surveys were completed at the time of urine collection. In the diary, daily defecation status and test article intake records were self-evaluated, together with stool volume (egg scale) and shape (six levels), stool odor (five levels), and the feeling of refreshment during defecation (three levels). The questionnaire comprised 21 items, 8 of which included the Japanese version of the Constipation Assessment Scale. ${ }^{29,30}$ Other questions were about skin condition, sleep quality, refreshment upon awakening, mood, appetite, and body condition, which were rated on a scale of five levels.

\section{Statistical Analysis}

A statistical analysis was performed to compare the evaluation items and determine their significance before and after intake of the test article. The paired $t$-test and the Wilcoxon signed-rank test were conducted on normally and non-normally distributed data, respectively. In the case of any sequential data with multiple steps, the Wilcoxon signed-rank test was conducted for the corresponding data. Bonferroni correction coefficient was used for multiple comparisons. The relationship between each data point was assessed using Pearson's correlation and Spearman's rank correlation coefficients with graphical inspection. Significant differences are indicated in the figures as ${ }^{*} p<0.05,{ }^{* *} p<0.01$, and ${ }^{* * *} p<0.001$.

\section{Results Study Participants}

Figure 1 shows a flowchart of the study participants. This study was aimed at Japanese adult women aged 30-50 years with a tendency to feel constipated. A total of 642 individuals applied, of which 60 participants who met the participation criteria underwent urinary 3-IS screening. The 30 applicants with the highest urinary 3-IS levels were selected for the study. The intake rate of the test article was $100 \%$ according to self-reporting; there were no omissions in the daily diary, questionnaires, or urine samples. Table 1 shows the background of the study participants. The subjects were women with an average age of $44.9 \pm 2.8$ years, and the frequency of self-reported defecation was about once per week in 9 (30\%) participants and once every 2 to 3 days in $21(70 \%)$ participants. The mean urinary 3-IS level before the intake of the test article was $141.0 \pm 60.9$ (mean \pm standard deviation [SD]) $\mu \mathrm{g} / \mathrm{mg} \mathrm{Cr}$ ), which was higher than the national average level $(45.1 \mu \mathrm{g} / \mathrm{mg} \mathrm{Cr})$.

\section{Changes in Urinary 3-IS}

Figure 2A shows changes from the initial urinary 3-IS level and how ingestion of the test article gradually decreased 


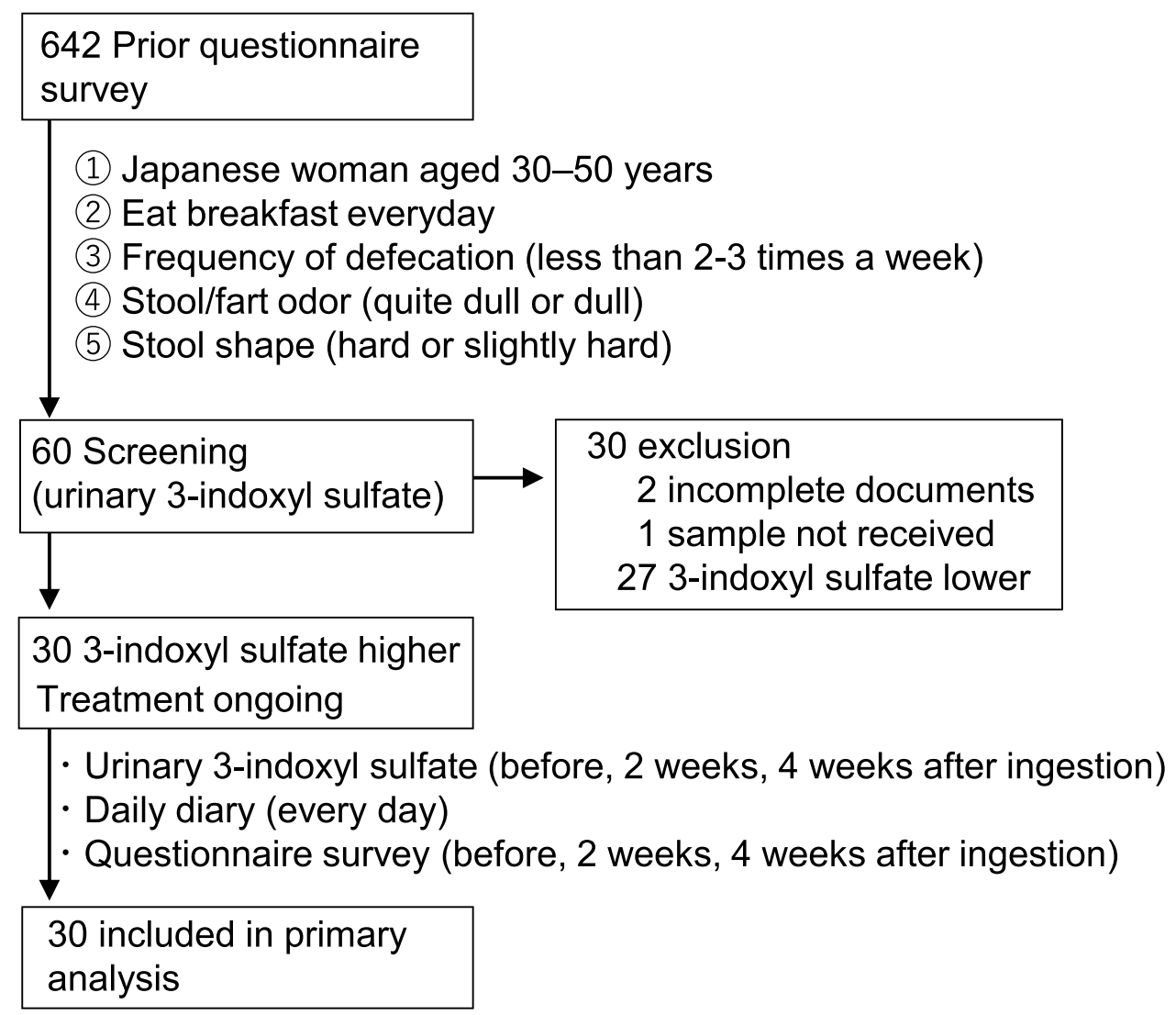

Figure I Trial participant tracking flowchart.

these levels. After 4 weeks, urinary 3-IS decreased 25\% from initial levels, which was statistically significant $(p<0.01)$. Changes in urinary 3-IS showed a high correlation to the initial 3-IS level (Figure 2B). Pearson's correlation coefficient was $0.74(p<0.001)$.

Furthermore, we analyzed changes in urinary 3-IS by categorizing the initial 3-IS levels (Table 2) into three groups: low level $(<100 \mu \mathrm{g} / \mathrm{mg} \mathrm{Cr} ; \mathrm{n}=8)$, middle level $(100-150 \mu \mathrm{g} / \mathrm{mg} \mathrm{Cr} ; \mathrm{n}=10)$, and high level $(>150 \mu \mathrm{g} / \mathrm{mg}$

Table I Profile of Participants Enrolled in This Study

\begin{tabular}{|l|l|}
\hline Item & Value \\
\hline Sex & Female \\
Age & $44.9(2.8)^{*}$ \\
Presence of pregnancy & None \\
Presence of chronic disease & None \\
Ingestion of medicine & None \\
Frequency of defecation (self- & $9(30 \%)$ once/week, 2I (70\%) \\
assessment) & once/2-3 days \\
Stool volume (self-assessment) & $2(6.7 \%)$ very few, 20 (66.7\%) few \\
Urinary 3-indoxyl sulfate level & $141.0(60.9)^{*} \mu g / m g$ creatinine \\
\hline
\end{tabular}

Note: *Values are presented as mean (SD) unless otherwise specified.
$\mathrm{Cr} ; \mathrm{n}=12$ ). In the high group, urinary 3-IS significantly decreased and the decrease observed at the fourth week was larger than that of the second week. In the middle group, a general decreasing trend was observed. However, in the low group, very little change was observed.

\section{Changes in the Indexes Related to Defecation}

From the questionnaire surveys completed during the test period, questions specific to the CAS were extracted and analyzed. It was observed that the CAS score had decreased by the second week after LEX ingestion and continued into the fourth week (Figure 3A). No significant changes were observed in all subjects in the number of bowel movements, the amount of defecation, and odor during defecation. This is not surprising as the average number of defecations per week from the start of the test was 5.87, which did not represent constipation tendencies.

Therefore, only those with constipation tendencies (defecation four times or less per week; $n=10$ ) were analyzed. Within this group, a significant increase in the number of defecations was observed. Similarly, regarding 

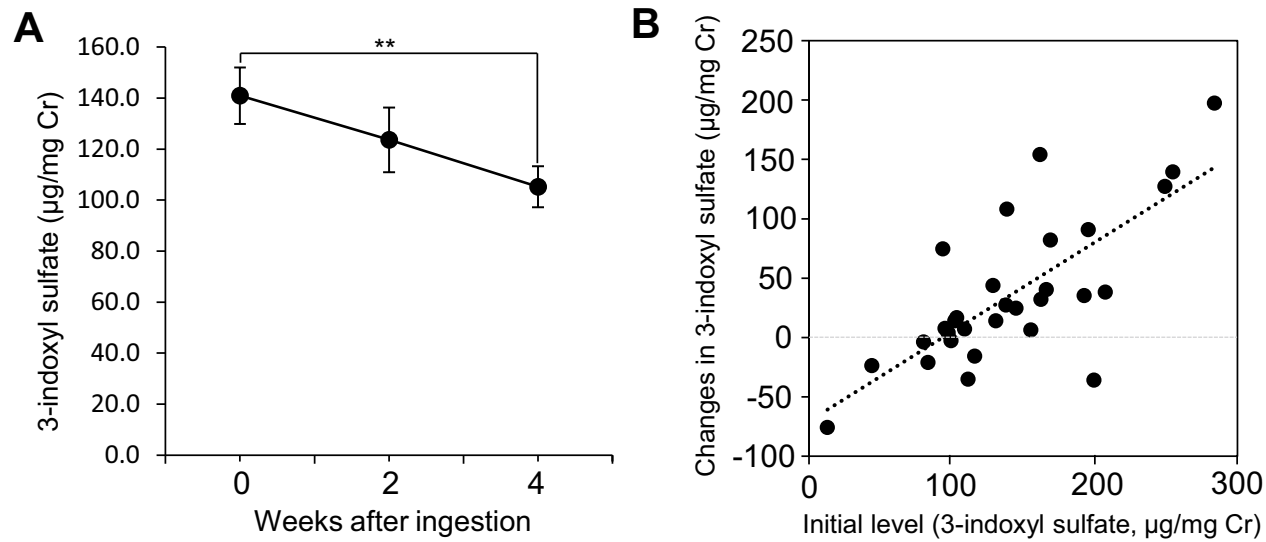

Figure 2 Changes in urinary 3-indoxyl sulfate levels.

Notes: $(\mathbf{A})$ Change with time from start of ingestion of the test article $(n=30)$. Statistical significance was determined using paired $t$-tests with Bonferroni correction coefficient. Significant differences from the initial values: ${ }^{* *} p<0.0$ I. Error bars indicate standard error. (B) Relationship between the initial urinary 3 -indoxyl sulfate levels and the amount of change. Pearson's correlation coefficient was $0.7407(p<0.001)$.

Abbreviation: $\mathrm{Cr}$, creatinine.

stool volume, when the single defecation volume was 1.5 or less, a significant increase in stool volume (single defecation) was observed between the first and fourth weeks. Lastly, a significant improvement was observed by those who had bad stool odor (Table 3 ).

\section{Changes in Other Physical Conditions}

During the test period, in addition to the abdominal symptoms questionnaire, we asked the participants to report on six items regarding skin condition, sleep quality, refreshment upon awakening, mood, appetite, and body tone on a scale of $1-5$. The total score of the six items statistically improved by the second week after ingestion of the test article and continued into the fourth week (Figure 3B). Of the six items, the scores for skin condition, sleep quality, and feeling of refreshment upon awakening in the morning significantly changed, and that for body tone also tended to change (Table 4).

\section{Correlation Between Urinary 3-IS and Physical Condition}

A modest degree of correlation between urinary 3-IS levels and overall physical condition, including CAS score, was observed (Figure 4A) for which the Pearson's correlation coefficient was $0.21(p<0.05)$. Moreover, a correlation between the scores for overall physical condition and the feeling of refreshment during defecation (Figure 4B) was observed in addition to a correlation between the single

Table 2 Hierarchical Analysis of Changes in 3-Indoxyl Sulfate Values from the Initial 3-Indoxyl Sulfate Values

\begin{tabular}{|c|c|c|c|c|c|}
\hline \multirow[t]{2}{*}{ Class *I } & \multirow[t]{2}{*}{ Week } & \multicolumn{2}{|c|}{$\begin{array}{l}\text { 3-Indoxyl Sulfate }(\mu \mathrm{g} / \mathrm{mg} \\
\text { creatinine) }\end{array}$} & \multirow[t]{2}{*}{$p$-value (Measured Value) vs $0 \mathrm{w}$} & \multirow[t]{2}{*}{$p$-value (Change Value) vs $<100$} \\
\hline & & $\begin{array}{l}\text { Measured } \\
\text { Value }\end{array}$ & $\begin{array}{l}\text { Change } \\
\text { Value }\end{array}$ & & \\
\hline$<100(n=8)$ & $\begin{array}{l}0 w \\
2 w \\
4 w\end{array}$ & $\begin{array}{l}75.84(10.00) \\
86.92(18.83) \\
80.99(9.74)\end{array}$ & $\begin{array}{l}- \\
\text { II.07 (I3.26) } \\
5.15(14.78)\end{array}$ & $\begin{array}{l}- \\
0.484 * 2 \\
0.575 * 2\end{array}$ & $\begin{array}{l}- \\
- \\
-\end{array}$ \\
\hline $100-150(n=10)$ & $\begin{array}{l}0 w \\
2 w \\
4 w\end{array}$ & $\begin{array}{l}122.35(5.58) \\
99.27(18.26) \\
101.87(11.38)\end{array}$ & $\begin{array}{l}- \\
-23.35(17.78) \\
-20.48(13.42)\end{array}$ & $\begin{array}{l}- \\
0.181 * 3 \\
0.122 * 3\end{array}$ & $\begin{array}{l}- \\
0.068 * 4 \\
0.192 * 5\end{array}$ \\
\hline$>150(n=12)$ & $\begin{array}{l}0 w \\
2 w \\
4 w\end{array}$ & $\begin{array}{l}199.86(14.16) \\
\text { I } 68.45(25.20) \\
\text { I24.27 (19.37) }\end{array}$ & $\begin{array}{l}- \\
-31.4 I(32.78) \\
-75.59(24.17)\end{array}$ & $\begin{array}{l}- \\
0.038 * 3 \\
0.007 * 3\end{array}$ & $\begin{array}{l}- \\
0.157 * 4 \\
0.008 * 5\end{array}$ \\
\hline
\end{tabular}

Notes: Values are presented as mean (SE) unless otherwise specified. $* 1$ classification by 3 -indoxyl sulfate initial value. $* 2$ Wilcoxon signed-rank test, $* 3$ paired $t$-test, $* 4$ Mann-Whitney $U$-test, $* 5$ unpaired $t$-test. 

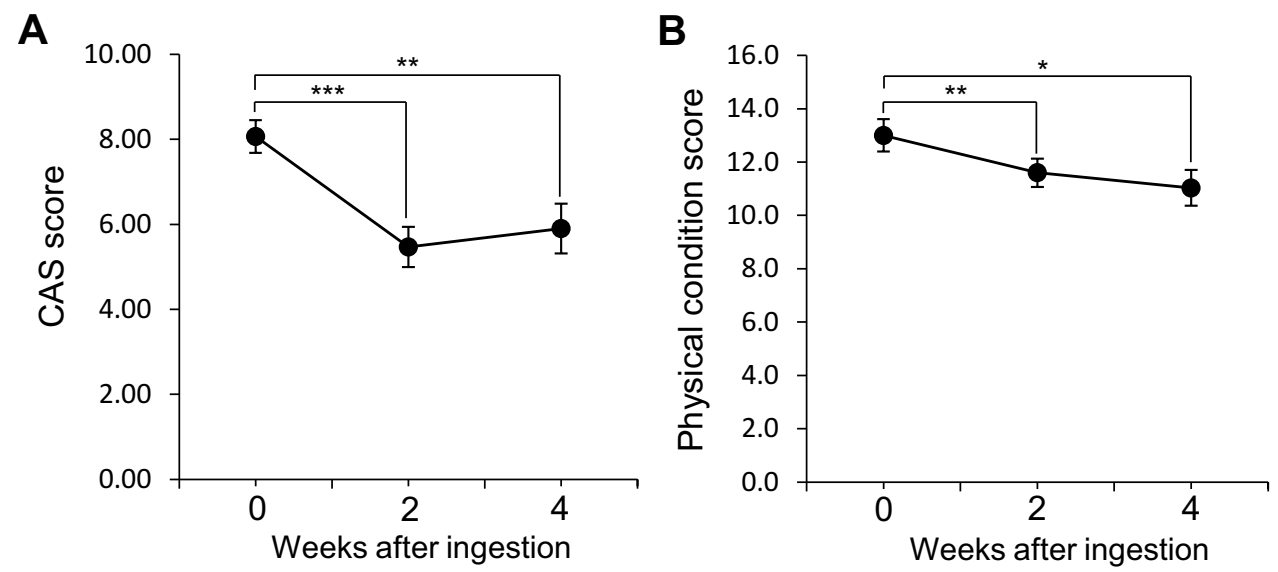

Figure 3 Changes in the constipation assessment scale and self-assessment of physical condition.

Notes: (A) CAS score. (B) Physical condition score. The score range is 0-24 points for the CAS and 0-30 points for the physical condition score, with lower scores being better. Statistical significance was determined using the Wilcoxon signed-rank test with Bonferroni correction coefficient. Significant differences from the initial values: $*_{p}<$ 0.05 , **p $<0.01$, *** $p<0.001$. $\mathrm{n}=30$.

Abbreviation: CAS, Constipation Assessment Scale.

defecation volume and the feeling of refreshment (Figure 4C). The Spearman's rank correlation coefficients were $0.31(p<0.01)$ and $0.51(p<0.001)$, respectively.

\section{Discussion}

To the best of our knowledge, this is the first study that assessed urinary 3-IS levels as an index of change in the intestinal environment in combination with LEX ingestion. This study showed that the regular ingestion of LEX resulted in an improvement in urinary 3-IS levels. The findings of this study add to existing evidence that LEX is beneficial for individuals with high 3-IS levels due to deterioration of their intestinal environment. However, since this was a pilot study and did not have a control, or placebo, group, the observed beneficial effects may be due to the placebo effect. Therefore, a study with a placebo or control group needs to be conducted. However, it is worth noting that the urinary 3-IS level correlated with the self-assessed physical condition index and that both the 3-IS level and the physical condition index improved due to the ingestion of LEX. While these correlations are very interesting, the results for this study only represent the effects on LEX on 30 women, and a more conclusive study would need a larger sample group with multiple genders.

Levels of urinary 3-IS are a simple and efficient screening tool for the presence of gut bacterial dysbiosis. Common microbes that have been found to affect urinary 3-IS test levels include Salmonella, Shigella, Campylobacter jejuni, Yersinia enterocolitica, Citrobacter freundii, Citrobacter diversus, Klebsiella pneumoniae, Pseudomonas aeruginosa,

Table 3 Changes in the Number of Defecations, Stool Volume, and Stool or Flatulence Odor During the Test Period

\begin{tabular}{|c|c|c|c|c|}
\hline \multirow[t]{2}{*}{ Item } & \multirow[t]{2}{*}{ Class } & \multicolumn{2}{|c|}{ Test Period } & \multirow[t]{2}{*}{$p$-value* } \\
\hline & & $0-I$ w & $3-4 w$ & \\
\hline \multirow[t]{3}{*}{ Number of defecations (per week) } & All $(n=30)$ & $5.87(0.50)$ & $6.00(0.49)$ & 0.663 \\
\hline & $\leqq 4(n=10)$ & $2.60(0.27)$ & $3.50(0.40)$ & 0.043 \\
\hline & $>4(n=20)$ & $7.50(0.35)$ & $7.25(0.5 \mathrm{I})$ & 0.485 \\
\hline \multirow[t]{3}{*}{ Single defecation volume (egg scale, weekly average) } & All $(n=30)$ & $1.89(0.14)$ & $2.16(0.21)$ & 0.107 \\
\hline & $\leqq 1.5(\mathrm{n}=12)$ & $1.19(0.10)$ & $1.53(0.16)$ & 0.037 \\
\hline & $>1.5(\mathrm{n}=18)$ & $2.37(0.12)$ & $2.58(0.19)$ & 0.379 \\
\hline \multirow[t]{4}{*}{ Odor evaluation scale } & All $(n=30)$ & $3.09(0.16)$ & $2.87(0.16)$ & 0.233 \\
\hline & $\geqq 3(n=19)$ & $3.56(0.15)$ & $3.16(0.17)$ & 0.017 \\
\hline & $\geqq 4(n=7)$ & $4.31(0.16)$ & $3.41(0.38)$ & 0.028 \\
\hline & $<3(n=11)$ & $2.27(0.17)$ & $2.37(0.39)$ & 0.386 \\
\hline
\end{tabular}

Notes: Values are presented as mean (SE) unless otherwise specified. *Wilcoxon signed-rank test. 
Table 4 Changes in Each Items of Phisical Condition During the Test Period

\begin{tabular}{|l|l|l|l|}
\hline \multirow{2}{*}{ Item } & \multicolumn{2}{|l|}{ Test Period } & \multirow{2}{*}{ p-value* } \\
\cline { 2 - 3 } & 0 Weeks & 4 Weeks & \\
\hline Skin condition & $2.47(0.14)$ & $2.03(0.17)$ & 0.013 \\
Quality of sleep & $2.37(0.13)$ & $1.87(0.15)$ & 0.004 \\
Morning awakening & $2.67(0.17)$ & $2.27(0.17)$ & 0.005 \\
Mood & $2.10(0.20)$ & $1.90(0.14)$ & 0.258 \\
Appetite & $1.00(0.14)$ & $0.90(0.13)$ & 0.445 \\
Tone of body & $2.40(0.18)$ & $2.07(0.16)$ & 0.068 \\
Total & $13.00(0.61)$ & $11.03(0.67)$ & 0.005 \\
\hline
\end{tabular}

Notes: Values are presented as mean (SE) unless otherwise specified. *Wilcoxon signed-rank test.

Staphylococcus aureus, some strains of Bacteroides and Escherichia coli, Clostridium difficile, Candida albicans, and other Candida species. This study did not directly analyze changes in gut microbiota composition; however, it is possible that these bacterial groups may have altered and thus affected changes in the metabolism of Trp. An analysis of change in intestinal bacteria would also be an important factor for a future study. In preliminary studies, ingesting LEX increased the amount of Bifidobacterium spp. and phyla Bacteroidetes and decreased the ratio of the phyla Firmicutes. $^{25}$ Another study observed a decrease in both the phenylalanine-derived 3-(3-hydroxyphenyl)-3-hydroxypropionic acid (HPHPA) as a urinary clostridial marker and Trp metabolites (5-hydroxyindoleacetic, quinolinic, and kynurenic acid) (unpublished data). ${ }^{31}$ From this, it can be suggested that ingesting LEX causes changes in the intestinal microbiota and affects Trp metabolism.

Trp not only functions as a nutrient enhancer but also plays an important role in balancing the intestinal tolerance of microbiota and their maintenance. Recent studies have underscored that changes in the microbiota modulate the host's immune system by controlling Trp metabolism. Bacterial Trp metabolites, such as indole and indoleic acid derivatives, can affect intestinal barrier integrity and immune
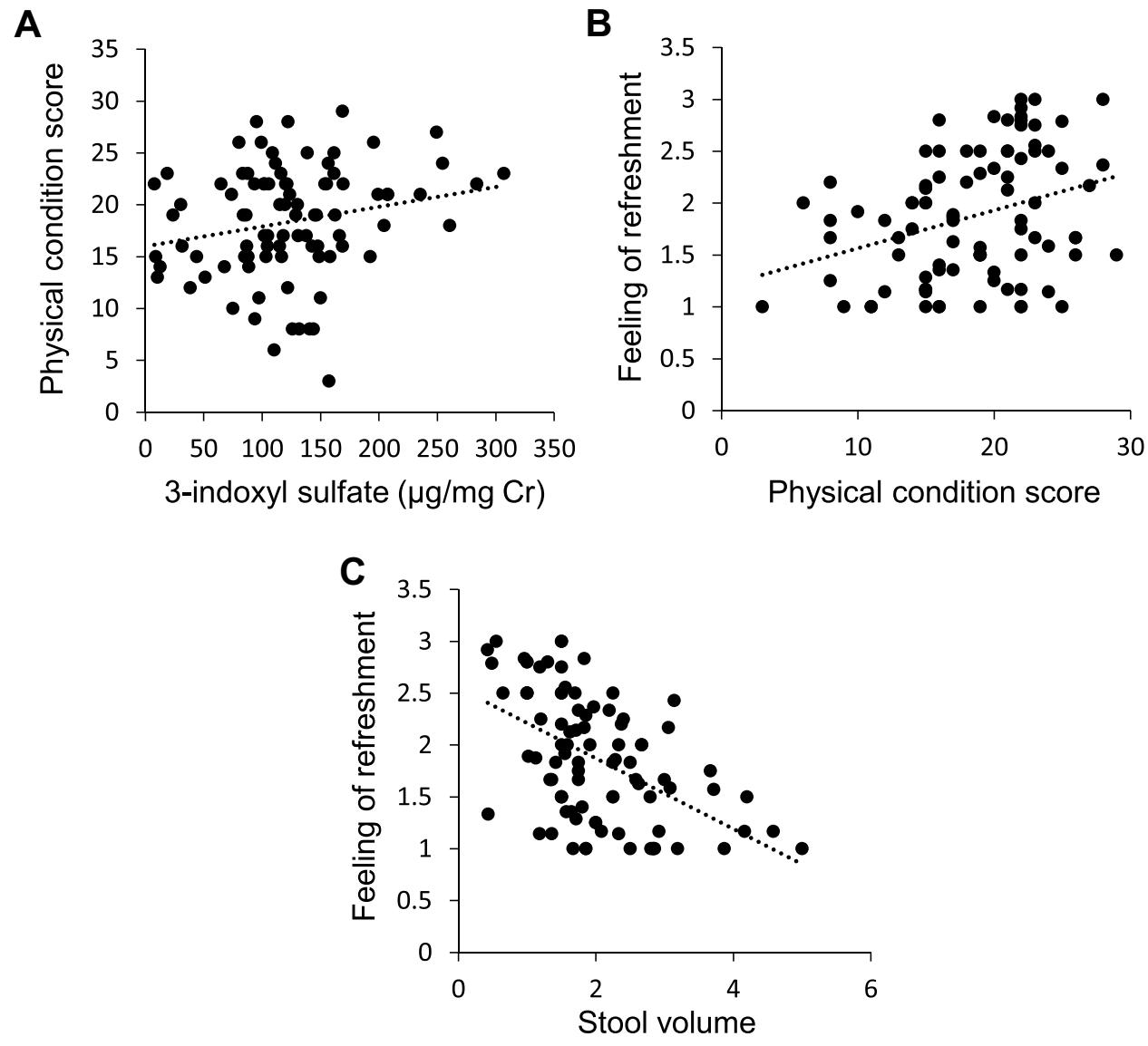

Figure 4 Correlation plot of several test parameters.

Notes: $(\mathbf{A})$ Urinary 3 -indoxyl sulfate versus self-assessment of physical condition, including CAS. Pearson's correlation coefficient was 0.2135 ( $p=0.043$ ). $(\mathbf{B})$ Selfassessment of physical condition within the CAS vs the feeling of refreshment during defecation. Spearman's rank correlation was 0.3120 ( $p=0.003)$. $(\mathbf{C})$ Stool volume (single defecation) vs the feeling of refreshment during defecation. Spearman's rank correlation coefficient was $0.5 \mathrm{I} 93(p=0.00 \mathrm{I})$.

Abbreviations: $\mathrm{Cr}$, creatinine; CAS, Constipation Assessment Scale. 
cells by activating pregnane $\mathrm{X}$ receptors (PXRs) or aryl hydrocarbon receptors (AhRs). ${ }^{32-34}$ Since the gut microbiota directly affects the types and levels of Trp metabolites, it subsequently regulates the mucosal immune response or mucosal integrity through AhRs or PXRs, respectively. In addition, bacterial Trp metabolites can regulate the production of IL-22, which plays a key role in intestinal homeostasis through AhRs. ${ }^{34}$

3-IS originates from indole catabolized by tryptophanase, which can be induced by Trp or repressed by glucose in most bacteria. Recently, indole has been recognized as a signaling molecule that regulates bacterial motility, biofilm formation, antibiotic resistance, and persister cell formation. Indole also affects virulence, which plays a role in affecting host cell invasion by other non-indole-producing species, such as Salmonella enterica and P. aeruginosa, and even the yeast $C$. albicans. ${ }^{35}$ Changes in urinary 3-IS due to the ingestion of LEX can therefore be considered changes in Trp metabolism, and various Trp metabolites, including indole, may directly or indirectly affect the intestinal tract.

3-IS is a typical uremic toxin and is normally excreted in urine. However, it can accumulate in blood if urine output slows due to decreased renal function. In fact, 3-IS is frequently implicated as a contributor to the progression of renal and vascular diseases. Studies have suggested that 3-IS also has adverse effects on bones, the central nervous system, and cardiovascular functions. ${ }^{36,37}$ Since the dialytic clearance of 3-IS is much lower than its kidney clearance, 3-IS accumulates in relatively high plasma levels in hemodialysis patients. In a small number of cases, the continuous ingestion of LEX by patients undergoing hemodialysis resulted in decreased blood 3-IS and blood urea nitrogen levels. ${ }^{38}$ The accumulation of 3-IS in blood depends on intestinal-derived indole, and the results of these patients are consistent with the results of this study. Thus, ingesting LEX may be effective for individuals with chronic renal failure.

\section{Conclusion}

In conclusion, oral ingestion of LEX for 4 weeks decreased urinary 3-IS levels in women with high urinary 3-IS levels and improved the defection status of constipated women. A correlation between the self-assessment of overall physical condition and urinary 3-IS level was also observed. The results indicated the efficacy of oral ingestion of LEX on the intestinal environment and the efficacy of monitoring urinary 3-IS to observe changes in the intestinal environment and physical condition.

\section{Acknowledgments}

We would like to thank all participants and applicants who took part in the study. We appreciate Healthcare Systems Co., Ltd. for their technical assistance in conducting urine analysis. Furthermore, we give special thanks to Mizukoshi R. and Sato T. (B\&S Corporation Co., Ltd.) for their assistance in setting up the test. Additionally, we would like to thank Enago for the English language review.

\section{Funding}

This study was funded by B\&S Corporation Co., Ltd.

\section{Disclosure}

$\mathrm{MF}$, RM, and YS receive compensation from B\&S Corporation Co., Ltd. TY and MM receive compensation from Healthcare Systems Co., Ltd. KF has no conflicts of interest directly relevant to the content of this article. The authors report no other conflicts of interest in this work.

\section{References}

1. Durack J, Lynch SV. The gut microbiome: relationships with disease and opportunities for therapy. $J$ Exp Med. 2019;216(1):20-40. doi: $10.1084 /$ jem.20180448

2. Qin J, Li R, Raes J, et al. A human gut microbial gene catalogue established by metagenomic sequencing. Nature. 2010;464 (7285):59-65. doi:10.1038/nature08821

3. Lozupone CA, Stombaugh JI, Gordon JI, Jansson JK, Knight R. Diversity, stability and resilience of the human gut microbiota. Nature. 2012;489(7415):220-230. doi:10.1038/nature11550

4. Ferguson JF, Allayee H, Gerszten RE, et al. Nutrigenomics, the microbiome, and gene-environment interactions: new directions in cardiovascular disease research, prevention, and treatment: a scientific statement from the American heart association. Circ Cardiovasc Genet. 2016;9(3):291-313. doi:10.1161/HCG.0000000000000030

5. Wang Z, Klipfell E, Bennett BJ, et al. Gut flora metabolism of phosphatidylcholine promotes cardiovascular disease. Nature. 2011;472(7341):57-63. doi:10.1038/nature09922

6. Hsiao EY, McBride SW, Hsien S, et al. Microbiota modulate behavioral and physiological abnormalities associated with neurodevelopmental disorders. Cell. 2013;155(7):1451-1463. doi:10.1016/j.cell.2013.11.024

7. Tang WH, Wang Z, Levison BS, et al. Intestinal microbial metabolism of phosphatidylcholine and cardiovascular risk. $N$ Engl J Med. 2013;368(17):1575-1584. doi:10.1056/NEJMoa1109400

8. Zhang LS, Davies SS. Microbial metabolism of dietary components to bioactive metabolites: opportunities for new therapeutic interventions. Genome Med. 2016;8(1):46. doi:10.1186/s13073-016-0296-x

9. Kirkland JL, Vargas E, Lye M. Indican excretion in the elderly. Postgrad Med J. 1983;59(697):717-719. doi:10.1136/pgmj.59.697.717

10. Bures J, Cyrany J, Kohoutova D, et al. Small intestinal bacterial overgrowth syndrome. World $J$ Gastroenterol. 2010;16 (24):2978-2990. doi:10.3748/wjg.v16.i24.2978

11. de Andrade LS, Ramos CI, Cuppari L. The cross-talk between the kidney and the gut: implications for chronic kidney disease. Nutrire. 2017;42(1):27. doi:10.1186/s41110-017-0054-x:

12. Greenberger NJ, Saegh S, Ruppert RD. Urine indicant excretion in malabsorptive disorders. Gastroenterology. 1968;55(2):204-211. doi:10.1016/S0016-5085(19)34071-5 
13. Tabaqchali S, Okubadejo OA, Neale G, Booth CC. Influence of abnormal bacterial flora on small intestinal function. Proc $R$ Soc Med. 1966;59(12):1244-1246.

14. Azhari AA. Beneficial role of lactic acid bacteria in food preservation and human health: a review. Res J Microbiol. 2010;5(12):1213-1221. doi: $10.3923 /$ jm.2010.1213.1221

15. Gilliland SE. Health and nutritional benefits from lactic acid bacteria. FEMS Microbiol Rev. 1990;7(1-2):175-188. doi:10.1111/j.15746968.1990.tb04887.x

16. Cavallini DC, Suzuki JY, Abdalla DS, et al. Influence of a probiotic soy product on fecal microbiota and its association with cardiovascular risk factors in an animal model. Lipids Health Dis. 2011;10 (1):126. doi:10.1186/1476-511X-10-126

17. Cheik NC, Rossi EA, Guerra RLF, et al. Effects of ferment soy product on the adipocyte area reduction and dyslipidemia control in hypercholesterolemic adult male rats. Lipids Health Dis. 2008;7 (1):50. doi:10.1186/1476-511X-7-50

18. Kitawaki R, Nishimura Y, Takagi N, Iwasaki M, Tsuzuki K, Fukuda M. Effects of lactobacillus fermented soymilk and soy yogurt on hepatic lipid accumulation in rats fed a cholesterol-free diet. Biosci Biotechnol Biochem. 2009;73(7):1484-1488. doi:10.1271/ bbb. 80753

19. Kobayashi M, Harada T, Takagi N, Tsuzuki K, Sugawara M, Fukuda M. Effect of lactic acid-fermented soymilk on lipid metabolism-related gene expression in rat liver. Biosci Biotechnol Biochem. 2012;76(1):19-24. doi:10.1271/bbb.100354

20. Shin R, Suzuki M, Mizutani T, Susa N. Improvement of experimentally induced hepatic and renal disorders in rats using lactic acid bacteria-fermented soybean extract (Biofermentics ${ }^{\mathrm{TM}}$ ). Evid Based Complement Alternat Med. 2009;6(3):357-363. doi:10.1093/ecam/ nem 126

21. Xiao CW. Health effects of soy protein and isoflavones in humans. J Nutr. 2008;138(6):1244S-1249S. doi:10.1093/jn/138.6.1244S

22. Fukui M, Fujino T, Tsutsui K, et al. The tumor-preventing effect of a mixture of several lactic acid bacteria on 1,2-dimethylhydrazineinduced colon carcinogenesis in mice. Oncol Rep. 2001;8 (5):1073-1078. doi:10.3892/or.8.5.1073

23. Takahashi S, Kawamura T, Kanda Y, et al. Activation of CD1d-independent NK1.1+ $\mathrm{T}$ cells in the large intestine by lactobacilli. Immunol Lett. 2006;102(1):74-78. doi:10.1016/j. imlet.2005.07.003

24. Odashiro K, Fukata M, Saito K, et al. The effects of lactic acid bacteria-fermented soymilk extract on patients with colonic polyps: a randomized, double-blind, placebo-controlled pilot trial. J Integr Stud Diet Habis. 2014;25(1):20-25. doi:10.2740/jisdh.25.20

25. Shima Y, Sasuga Y. Effects of lactic acid bacteria-fermented soymilk extract on intestinal microflora: an open pilot study on human volunteer. J Jpn Mibyo Sys Assoc. 2014;20(1):71-75.
26. Yoshimine M, Kamoi H, Yonekura K, Sasuga Y, Kamoi K. Effects of extract obtained from lactic-acid-producing bacteria throughout periodontitis. Nihon Shishubyo Gakkai Kaishi. 2018;60(3):139-146. doi: 10.2329 /perio.60.139

27. Aguilar-Toalá JE, Garcia-Varela R, Garcia HS, et al. Postbiotics: an evolving term within the functional foods field. Trends Food Sci Tech. 2018;75:105-114. doi:10.1016/j.tifs.2018.03.009

28. Wegh CAM, Geerlings SY, Knol J, Roeselers G, Belzer C. Postbiotics and their potential applications in early life nutrition and beyond. Int J Mol Sci. 2019;20(19):4673. doi:10.3390/ijms20194673

29. Kira I. Validity and reliability of a Japanese version of the patient assessment of constipation quality of life questionnaire. Kango Kenkyu. 2013;36(2):119-127. doi:10.15065/jjsnr.20130421012

30. McMillan SC, Williams FA. Validity and reliability of the constipation assessment scale. Cancer Nurs. 1989;12(3):183-188. doi:10.1097/00002820-198906000-00012

31. Shaw W. Increased urinary excretion of a 3-(3-hydroxyphenyl)3-hydroxypropionic acid (HPHPA), an abnormal phenylalanine metabolite of Clostridia spp. in the gastrointestinal tract, in urine samples from patients with autism and schizophrenia. Nutr Neurosci. 2010;13 (3):135-143. doi:10.1179/147683010X12611460763968

32. Gao J, Xu K, Liu H, et al. Impact of the gut microbiota on intestinal immunity mediated by tryptophan metabolism. Front Cell Infect Microbiol. 2018;8:13. doi:10.3389/fcimb.2018.00013

33. Venkatesh M, Mukherjee S, Wang H, et al. Symbiotic bacterial metabolites regulate gastrointestinal barrier function via the xenobiotic sensor PXR and toll-like receptor 4. Immunity. 2014;41 (2):296-310. doi:10.1016/j.immuni.2014.06.014

34. Zelante T, Iannitti RG, Cunha C, et al. Tryptophan catabolites from microbiota engage aryl hydrocarbon receptor and balance mucosal reactivity via interleukin-22. Immunity. 2013;39(2):372-385. doi:10.1016/j.immuni.2013.08.003

35. Li G, Young KD. Indole production by the tryptophanase TnaA in Escherichia coli is determined by the amount of exogenous tryptophan. Microbiology. 2013;159(2):402-410. doi:10.1099/ mic. 0.064139-0

36. Leong SC, Sirich TL. Indoxyl sulfate-review of toxicity and therapeutic strategies. Toxins (Basel). 2016;8(12):358. doi:10.3390/ toxins 8120358

37. Liu WC, Tomino Y, Lu KC. Impacts of indoxyl sulfate and p-cresol sulfate on chronic kidney disease and mitigating effects of AST-120. Toxins (Basel). 2018;10(9):367. doi:10.3390/toxins10090367

38. Kawashima A, Mimuro T, Matsuue K, Nitta K. Effects of probiotics on blood indoxyl sulfate in patients on maintenance dialysis. Japan Soc Nephrol. 2006;48(3):280. doi:10.14842/jpnjnephrol1959.48.234
Nutrition and Dietary Supplements

\section{Publish your work in this journal}

Nutrition and Dietary Supplements is an international, peerreviewed, open access journal focusing on research into nutritiona requirements in health and disease, impact on metabolism and the identification and optimal use of dietary strategies and supplements necessary for normal growth and development. The journal welcomes submitted papers covering original research, basic science, clinical \& epidemiological studies, reviews and evaluations, guidelines, expert opinion and commentary, case reports and extended reports. The manuscript management system is completely online and includes a very quick and fair peer-review system, which is all easy to use. Visit http://www.dovepress.com/testimonials.php to read real quotes from published authors. 